\title{
A Comprehensive Management of Complicated Anterior Maxillary Crown Fracture in a 15-year-old Adolescent
}

\author{
Pallawi $^{1}$, Chitrita G Mukherjee ${ }^{2}$, Siddharth Anand ${ }^{3}$, Rajat K Singh ${ }^{4}$, Bidyut Seal ${ }^{5}$
}

\begin{abstract}
Dental trauma to anterior maxillary teeth is a frequent incidence in young patients. Pediatric dentists have to deal with such dental traumatic injuries on a regular basis in their daily routine practice. Several studies have reported reattachment of traumatized fractured tooth segment with or without post placement using dentine bonding agent and adhesive resin cement. This case report presents a comprehensive and multidisciplinary management of maxillary anterior teeth with complicated crown fracture and its reattachment with prefabricated glassreinforced composite fiber post and dual-cure adhesive resin followed by a permanent restoration.

Keywords: Adhesive resins, Complicated crown fracture, Glass-reinforced composite fiber post, Permanent restoration, Reattachment. International Journal of Clinical Pediatric Dentistry (2020): 10.5005/jp-journals-10005-1763
\end{abstract}

\section{INTRODUCTION}

Coronal fractures of anterior tooth in children and adolescents are very common. Maxillary central incisors are prone to traumatic dental injuries, whereas maxillary lateral incisors and mandibular central incisors are less frequently involved. ${ }^{1}$ These dental injuries are prevalent in clinical practice and their management is a challenge for the clinician, who, depending on the type of injury, has to choose among several possibilities to approach the problem. The treatment options should aim to save the traumatized tooth which requires a quick functional and Esthetic repair and can be saved primarily by an endodontic therapy followed by composite buildup, cast post, prefabricated post, veneering, and full coverage crowns for functional and esthetic purposes.

When there is minimal violation of biological width in coronal tooth fractures, it is better to reattach the tooth fragment. ${ }^{1}$ This simple procedure can provide a better core for full coverage crown that results in a positive psychological response of the patient and restores function with good and long-lasting esthetics. One of the concerns about reattachment of fractured tooth segment is the fracture strength of the restored tooth and its longevity. ${ }^{2}$ However, reattachment procedures serves only as a semi-permanent replacement and the longevity is questionable in comparison with the conventional approaches. ${ }^{3}$

The purpose of this case report is to present a case of complicated maxillary coronal fracture with endodontic treatment and reattachment of fractured segment that provides a central support stump for restoration of dental morphology followed by porcelain-fused metal crown for longevity and to avoid future possible malfunction.

\section{Case Description}

A female patient aged 15 years with her mother reported to outpatient department of pedodontics and preventive dentistry with complaint of broken teeth, pain, and swelling in the upper front region of the jaw since 2 days. Detailed history revealed that patient had fallen on hand pump while playing during school hours. Pain was dull and continuous in nature that subsided on taking medication whereas swelling was diffuse and limited to the upper front region of the lip. On extraoral examination, the soft tissue

\begin{abstract}
${ }^{1-5}$ Department of Pedodontics and Preventive Dentistry, Buddha Institute of Dental Sciences and Hospital, Patna, Bihar, India

Corresponding Author: Siddharth Anand, Department of Pedodontics and Preventive Dentistry, Buddha Institute of Dental Sciences and Hospital, Patna, Bihar, India, Phone: +91 8409171918, e-mail: drsiddharthanand@yahoo.co.in

How to cite this article: Pallawi, Mukherjee CG, Anand S, et al. A Comprehensive Management of Complicated Anterior Maxillary Crown Fracture in a 15-year-old Adolescent. Int J Clin Pediatr Dent 2020;13(3):303-305.
\end{abstract}

Source of support: Nil

Conflict of interest: None

laceration was present on upper and lower parts of the lip, whereas intraoral examination revealed the presence of laceration on labial mucosa, complicated crown fracture (Ellis class III) with 11 and Ellis class II with 21. The fractured segment of crown with 11 was loosely attached to the palatal gingiva. Intraoral periapical radiograph showed the widening of periodontal ligament with respect to 11 and 21. Clinical and radiographic examination confirmed the Ellis classes III and II fractures with 11 and 21, respectively (Fig. 1).

The patient and her mother were explained about the treatment procedures in detail and written consent was obtained. Single sitting root canal treatment and reattachment of fractured crown fragment with 11 were planned along with antibiotics and analgesics. Prior to this, oral prophylaxis was done. Under aseptic conditions, local anesthesia was administered in the maxillary central incisors region and access opening was carried out with respect to 11 , pulp was extirpated and the canal was thoroughly prepared followed by obturation with gutta-percha (Fig. 2). The broken fragment was approximated intraorally for the evaluation of proper positioning of fractured coronal structure, and beveling was done on the fractured fragment and the tooth to increase the retention. The dentin and enamel of tooth as well as fractured segment were acid etched with $37 \%$ phosphoric acid (3M ESPE; Scotchbond ${ }^{\mathrm{TM}}$ Multipurpose Etchant, USA) for 20 seconds followed by through rinsing for 10 seconds 


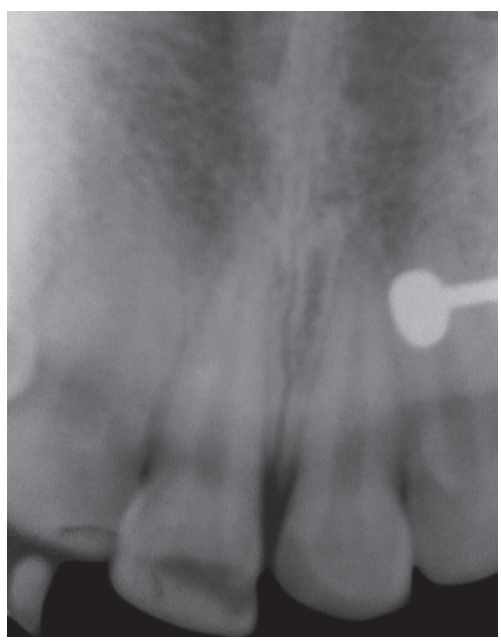

Fig. 1: Preoperative IOPA

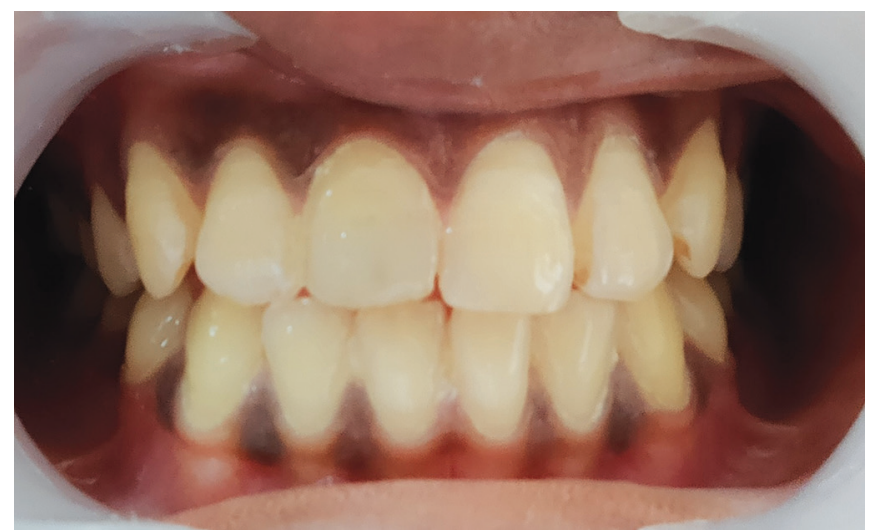

Fig. 3: Reattachment of broken segment

and drying. Bonding agent (3M ESPE; Scotchbond ${ }^{\mathrm{TM}}$ ) was applied and light cured according to the manufacturer's guidelines. Then fractured segment was accurately reattached to its proper position followed by finishing and polishing (Fig. 3). Occlusion clearance was carefully checked and postoperative instructions were given in detail.

The patient was recalled after 3 months, and she presented with detached tooth fragment with 11. Therefore, comprehensive decision was taken to reattach the fragment with glass fiberreinforced composite post followed by porcelain fused to metal crown. The fractured tooth segment was stored in $10 \%$ formalin till the completion of procedure. Root canal and fractured segment were prepared for adaptation of fiber post-reinforced composite (Tenax Fiber Trans; Coltene Whaledent Pvt. Ltd, USA) followed by etching with $37 \%$ phosphoric acid (3M ESPE; Scotchbond ${ }^{\mathrm{TM}}$ ) to the pulpal wall of the root and fractured crown and then bonded with dual-cure resin (3M ESPE; Rely X ${ }^{\mathrm{TM}}$ U200, Germany) under visible light (Fig. 4). The occlusal clearance was evaluated and post instructions were given. The patient was evaluated after 7 days and the tooth 11 was prepared for full coverage crown, i.e., porcelain fused to metal crown. The tooth were temporary and permanent crown were cemented after 2 days using glass ionomer luting cement (3M ESPE; Ketac ${ }^{T M}$ Cem Radiopaque, Germany; Fig. 5). The patient was recalled

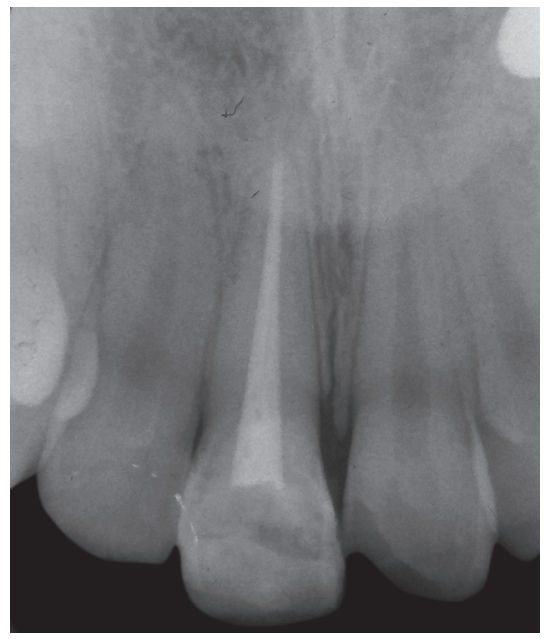

Fig. 2: GP obturation

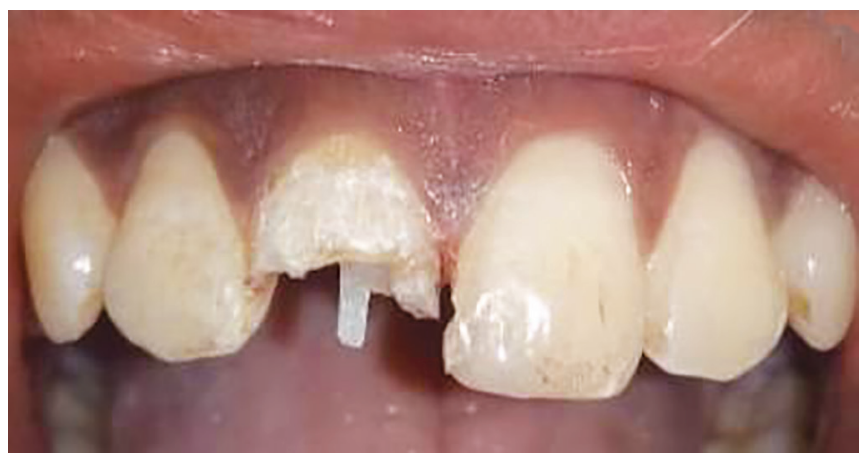

Fig. 4: Fiber post adaptation

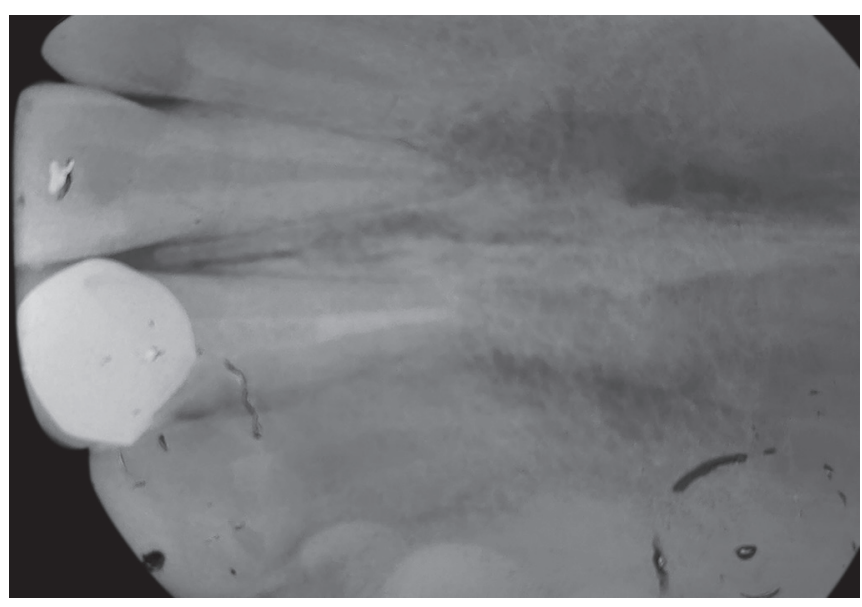

Fig. 5: After PFM crown delivery

after 2 weeks for routine checkup; and further postoperative radiograph after 1 month, 6 months, and 12 months showed no pathology with the treated teeth (Fig. 6).

\section{Discussion}

Traumatic fracture of anterior teeth is an awful experience for the children and young adolescents that requires immediate treatment 


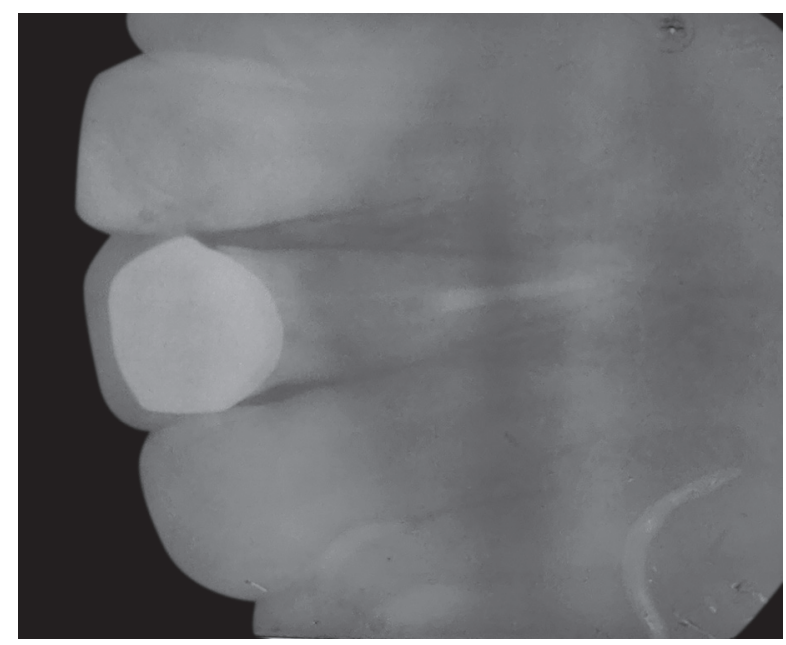

Fig. 6: Follow-up at 6 months

as it not only damages the dentition, but it also causes long-term psychological effect on the child and the child's parents. ${ }^{4}$ It has been reported that there is a positive social and emotional response from the patient as well as their parents to the conservation of natural tooth structure. ${ }^{5}$ Esthetic, functional, and biologic restoration of the fractured tooth segment is an intimidating challenge for a clinician.

Results of conventional composite resin restoration may lead to less incisal translucency, ideal contours, and color match. The tooth buildup with composite resins tends to take up stains from food and beverages that a patient consumes over a period of time and thus the esthetic is compromised. ${ }^{3}$ All restorative techniques, however, present limitations and reattachment is not an exception. The first limitation is that the longevity of this procedure is not predictable and the fragment may detach from the remaining tooth tissue.

The attachment of the fragment in an adequate position may be difficult depending on the characteristics of the fracture and fragment. Esthetics is a challenging task for nonvital tooth as pulpless tooth loses its brightness and translucency overtime and may not retain the original hue and translucency, resulting in poor esthetics. ${ }^{1,6}$ The bonding between the fractured fragment and the remaining tooth structure may also present a demarcating line with a different shade. Long-term prognosis of the fractured segment may be queried and may thus require other restorative alternatives such as veneering and crown fabrication in case of failure.

To overcome such problem, porcelain-fused metal crown was selected as a permanent restoration. ${ }^{7,8}$ Combined techniques as in this case report can be used in the management of complicated anterior tooth fracture that has shown better prognosis, functions, esthetics, and satisfaction of patient and parent. In cases of pulpal involvement, a root post is mainly followed after endodontic treatment to provide mechanical support for the fractured segment capable of resisting occlusal loads which simplifies and shortens the clinical procedure. ${ }^{9,10}$

In present case, glass fiber-reinforced composite post and dual-cure luting adhesive composite system was used followed by cast restoration which provided better esthetics and longevity of a non-vital fractured tooth.

\section{Conclusion}

This case report describes multidisciplinary comprehensive management of a complicated crown fracture, resulting in preservation of original tooth structure followed by permanent restoration.

\section{References}

1. Macedo GV, Diaz PI, Fernandes AC, et al. Reattachment of anterior teeth fragments: a conservative approach. J Esthet Restor Dent 2008;20(1):5-20. DOI: 10.1111/j.1708-8240.2008.00142.x.

2. Jyothi M, Jyothirmayi BSL, Sirisha K, et al. Reattachment- conservative management of complicated crown fractures in anterior teeth. Int J App Dent Sci 2016;2(1):10-13.

3. Anand S, Hegde DY, Yeluri R, et al. Comprehensive management of complicated crown fracture in a 10-year-old child. Int J Pedod Rehab 2017;2(1):46-50. DOI: 10.4103/ijpr.ijpr_1_17.

4. Hegede RJ. Tooth fragment reattachment - an esthetic alternative: report of a case. J Indian Soc Pedod Prev Dent 2003;21:117-119.

5. Saha R. Reattachment of fractured anterior tooth: a case report. Int J Dent Med Res 2015;1(6):113-115.

6. Mojirade AD, Funmilayo A-SIM, Olaide GS. Reattachment of fractured anterior tooth: a 2-year review of a case. Int J Prosthod Restorat Dent 2011;1(2):123-127. DOI: 10.5005/jp-journals-10019-1023.

7. Jain V, Gupta R, Duggal R, et al. Restoration of traumatized anterior teeth by interdisciplinary approach: report of three cases. J Ind Soc Pedod Prev Dent 2005;23(4):193-197. DOI: 10.4103/0970-4388.19009.

8. Joshi SR, Pendyala GS, Saraf V, et al. A comprehensive oral and dental management of an epileptic and intellectually deteriorated adolescent. Dent Res J 2013;10(4):562-567.

9. Freedman GA. Esthetic post-and-core treatment. Dent Clin North Am 2001;45(1):103-116.

10. Koutayas SO, Kern M. All-ceramic posts and cores: the state of the art. Quintessence Int 1999;30(6):383-392. 